\title{
Experimental Study on Deflagration-to-Detonation Transition Enhancement Methods in a PDE
}

\author{
T. H. New ${ }^{1}$, P. K. Panicker ${ }^{2}$, K. F. Chui ${ }^{3}$, H. M. Tsai ${ }^{4}$ and F. K. Lu ${ }^{5}$ \\ ${ }^{1}$ Department of Engineering, University of Liverpool, Brownlow Hill, L69 3GH, UK \\ ${ }^{3,4}$ Temasek Laboratories, National University of Singapore, 10 Kent Ridge Crescent, Singapore 119260 \\ ${ }^{2,5}$ Aerodynamics Research Center, Mechanical and Aerospace Engineering Department \\ University of Texas at Arlington, Arlington, TX 76019, USA
}

\begin{abstract}
An experimental investigation was carried out to study the performance of a pulse detonation engine platform incorporating commercial, off-the-shelf solenoid valve gas injectors and non-conventional deflagration-to-detonation transition enhancing devices. The study made use of stoichiometric propane-oxygen mixtures with low-energy ignition sources. The gas injectors were observed to be sufficiently robust and operated reliably under the high working temperature and pressure conditions normally associated with pulsed detonation operations provided appropriate preventive measures were taken. One of the major motivations in utilizing these gas injectors lies in the ease and accuracy in controlling their injection operations electronically, which allows for tight integration with auxiliary electronic control and measurement systems. This paper reports on the initial success of integrating these gas injectors into a moderate-frequency pulsed detonation engine system as well as the effectiveness of the deflagration-to-detonation transition enhancing devices which included Shchelkin spiral, circumferential and helical grooves, as well as convergentdivergent throats. Lastly, operational insights in the practical use of gas injectors and the impact on pulse detonation operations are highlighted.
\end{abstract}

\section{Introduction}

$\mathrm{P}$ ULSE detonation engines have been extensively studied experimentally, numerically and analytically ${ }^{1-9}$ over the past two decades and have been shown to be able to offer potentially better performance over conventional aeropropulsion platforms in terms of propulsive thrust and operating efficiencies, while achieving robustness and cost effectiveness. Unlike existing turbofan or turbojet engines which possess highly intricate and complex arrangements of compressor, combustion and turbine stages, a pulse detonation engine (PDE) attempts to make use of highfrequency pulsed detonations to provide a non-conventional but highly effective technique for producing aeropropulsive thrust. In contrast to the constant pressure combustion processes in a conventional turbine engine, a PDE is able to produce quasi-steady propulsive thrust through high frequency, constant volume, cyclical detonations.

One of the biggest advantages of utilizing detonations for thrust production is the possible elimination of compressor and turbine stages since detonations, by themselves, will produce very high compression levels, thereby eliminating the heavy and complex turbomachinery components. By eliminating a significant number of turbopumps, compressor and turbine stages, a PDE can drastically reduce the overall engine weight as well as the level of complexity associated with the vast number of moving parts existing in conventional turbine engines. Furthermore, using pulsed detonations to produce thrust is attractive because of the associated high cycle performance and efficiency, as demonstrated by earlier thermodynamic cycle analyses. ${ }^{10-13}$

\footnotetext{
${ }^{1}$ Lecturer, Department of Engineering, University of Liverpool, UK

${ }^{2}$ Graduate Research Associate, Aerodynamics Research Centre, University of Texas at Arlington, Student Member AIAA

${ }^{3}$ Associate Scientist, Temasek Laboratories, National University of Singapore

${ }^{4}$ Principal Research Scientist, Temasek Laboratories, National University of Singapore

${ }^{5}$ Professor, University of Texas at Arlington, Associate Fellow AIAA
} 
Despite the advances made in developing pulse detonation engines, many fundamental scientific questions and technological problems remain unresolved. In particular, the question of how to achieve high-frequency detonations successfully and reliably using the shortest detonation tube possible is still a recurring but yet important research theme. To begin to understand what are the scientific and technical challenges facing that question, it has to be noted that the issues actually consist of two separate components. First is the issue of high-frequency ignition and injection of fuel-oxidizer mixture. Secondly is the challenge of achieving detonations in the shortest distances possible after ignition. Injection of fuel and oxidizer is typically carried out using mechanical valves, such as rotary valves, or using high-speed solenoid valves. Although rotary valve systems offer design and operational simplicity, there remain operational issues regarding precise control of the injection volumes and the maximum frequency at which these valves can operate. On the other hand, while custom-designed high-speed solenoid valves are able to reach very high operating frequencies, their cost may be prohibitive due to the special built-in protections for tolerance against high working pressures and temperatures for detonation operations.

As for the challenge of achieving successful detonations within a short distance after ignition, several solutions have been proposed to resolve the issue. First, detonations can be directly initiated by using high-energy electrical ignition although this will entail the use of an elaborate and usually bulky ignition system. Furthermore, highfrequency high-energy ignition may not be feasible for prolonged operation as the electrodes may not be able to withstand the rapid buildup of heat and the repeated strong shock loading. Secondly, a hybrid ignition system may be used such that a small amount of easily detonable, primary fuel-oxidizer mixture is detonated before allowing the detonation front to continue propagating into a larger amount of less detonable secondary fuel-oxidizer mixture. The detonation front resulting from the primary fuel-oxidizer mixture will then have sufficient energy to initiate and sustain continuing detonation through the secondary fuel-oxidizer mixture. However, the complexity involved in regulating and using two different fuel-oxidizer mixtures as compared to other techniques is significant and may not be suitable for all PDE applications. Thirdly, detonations can result from deflagrations initiated via a low-energy ignition in conjunction with deflagration-to-detonation transition (DDT) enhancing devices along the deflagration path. Numerous investigators have shown that these devices can reduce the DDT distance and time significantly, ${ }^{14-}$ ${ }^{17}$ notably Shchelkin who pioneered the use of helical spirals along the bore of the detonation tube to promote DDT. The general consensus is that two- and three-dimensional profiled obstacles located regularly within the detonation tube are able to promote flame turbulence and accelerate the flame velocities such that the flame fronts eventually coincide with the shock fronts to form successful detonation fronts. The use of detonation-enhancing devices is by far one of simplest and effective technique to produce detonations within the short distances required in a practical PDE.

The brief review above leads to the two main objectives in this experimental investigation. First, the investigation is to explore the feasibility of incorporating commercial, off-the-shelf gas injectors meant for internal combustion engines into a PDE platform. Secondly, the effectiveness of non-conventional DDT enhancing devices using such a platform is explored. As an initial step to assess the feasibility of using these gas injectors for PDE applications, emphasis was placed on the successful integration of the gas injectors and associated system controls rather than achieving as high a frequency as possible (which will be addressed in another study). The decision to use commercial gas injectors was due to several considerations. First is their significantly lower cost than customdesigned gas injectors. Secondly, these injectors are much smaller, lighter and faster than rotary valves. Lastly, these injectors can be integrated into electronic control systems with high timing accuracy. On the other hand, though, these injectors offer lower levels of protection against high working temperatures and pressures which required preventive measures. Custom-designed gas injector housing, check valves and water-cooling subsystems have to be used to ensure safe and reliable operations of the gas injectors, which will be elaborated in the next section.

Another important objective of the investigation is to study the effectiveness of several non-conventional DDTenhancing configurations, while benchmarking them against the well-established Shchelkin spiral. The investigated configurations included conical, convergent-divergent throats with different convergent-divergent angles, parallel grooves and helical grooves. The effectiveness of these devices was characterized in terms of the detonation pressure profiles, time-of-flight velocities and thrust measurements. In the next section, an extensive description of the experimental setup, instrumentation and measurement methodologies will be given. Subsequently, some of the significant experimental results obtained will be reported and analyzed in Section III. Lastly, the overall implementation and results obtained from the experimental investigation and future directions where further studies can be undertaken will be concluded in Section V. 


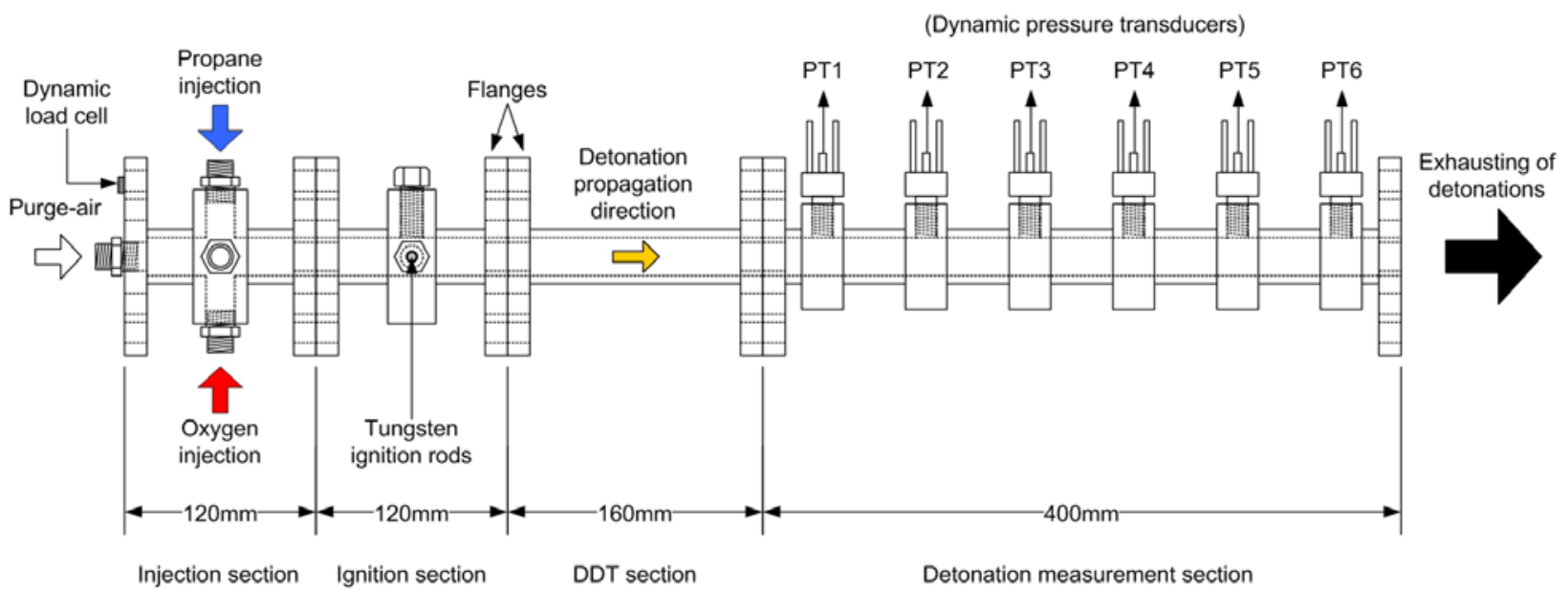

Fig. 1 Schematics of the PDE platform.

\section{Experimental setup}

\section{A. Detonation tube}

The main detonation tube was fabricated from ASME Schedule 80 stainless steel pipe with an inner diameter of $24.3 \mathrm{~mm}$ and an outer diameter of $33.4 \mathrm{~mm}$. The tube is made of four detachable sections which have standard high-pressure flanges fully welded to their ends. These four sections are the injection section, ignition section, DDT section and the detonation measurement section as shown schematically in Fig. 1. The $120 \mathrm{~mm}$ long injection section housed three $12.7 \mathrm{~mm}$ (0.5 in.) diameter injection ports for introducing propane, oxygen and purge air . To improve mixing, the oxygen and propane injection ports were arranged such that the gas jets impinged upon each other. As for the purge air, its injection port was located at the end of the injection section along the detonation propagation direction to facilitate purging of the detonation products. For the $120 \mathrm{~mm}$ ignition section, two ports were available for inserting two 5/32 in.-diameter lanthanated tungsten rods that served as electrodes for a lowenergy igniter. These rods were encased in ceramic tubes to provide electrical insulation. Moreover, these rods were connected to a commercial, off-the-shelf automotive ignition system rated at approximately $150 \mathrm{~mJ}$ per ignition spark. Such an electrode setup was preferred over using conventional automotive spark plugs since these have energy limiting, high resistance resistors used to reduce electromagnetic interference. However, significant electromagnetic noise detected in an earlier study ${ }^{17}$ meant that a compromise between energy output and electromagnetic interference reduction still had to be made by using a $3 \mathrm{k} \Omega$ resistor in series with the electrode setup. Nonetheless, higher energy output can be obtained compared to conventional automotive plugs. It is worth noting that the gap between the two tungsten rod ends within the ignition section can be varied manually prior to an experiment to achieve good sparking. The $160 \mathrm{~mm}$ long DDT section was used for testing non-conventional DDT configurations. Lastly, dynamic pressure transducers were located $65 \mathrm{~mm}$ apart from each other in the $800 \mathrm{~mm}$ long detonation measurement section. When fully assembled, the entire detonation tube measured approximately 800 $\mathrm{mm}$, giving a length-to-diameter ratio close to 33. For safety precautions, the detonations exhausting from the detonation tube were directed into a $254 \mathrm{~mm}$ diameter hollow metal pipe with baffles and fitted with a venturi fan driven by compressed air to flush any potential propane or oxygen remnants.

\section{B. Gas injectors}

Commercially available off-the-shelf gas injectors with a nominal working pressure of $550 \mathrm{kPa}$ (AFS Gs60-055-c series, Alternative Fuel Systems Inc., Calgary, Canada) were used in the present study for precise control over the timing and injection volumes of propane and oxygen. The gas injectors were driven by an AFS injector driver module which takes in and converts standard TTL signals into appropriate control currents to operate the high-speed solenoids in the gas injectors yielding typical response times of less than $2 \mathrm{~ms}$ with 8 A peak and 2 A holding currents at 12 VDC. The above characteristics make these injectors ideal for high-frequency gas injection. Although the injectors were capable of operating at a frequency of up to $50 \mathrm{~Hz}$, only moderate firing frequencies of approximately $15 \mathrm{~Hz}$ were realized during this initial feasibility study. However, critical operating knowledge derived from this study has led to on-going attempts to increase the firing frequency significantly by improving the 


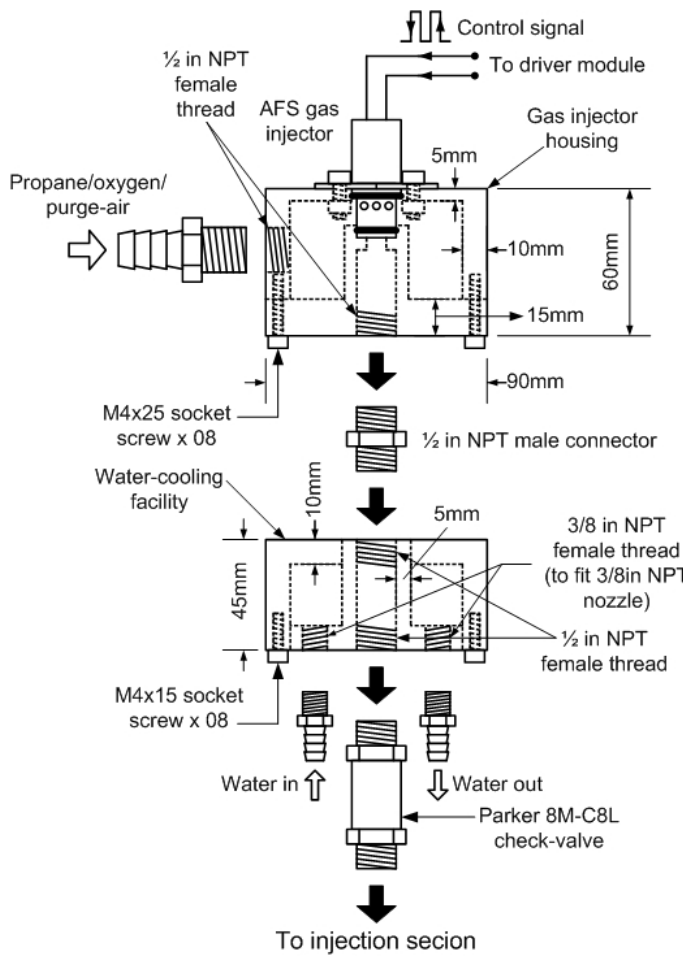

Fig. 2 Schematic of the custom-designed gas injector housing incorporating safety features such as check valves and water-cooling subsystems.

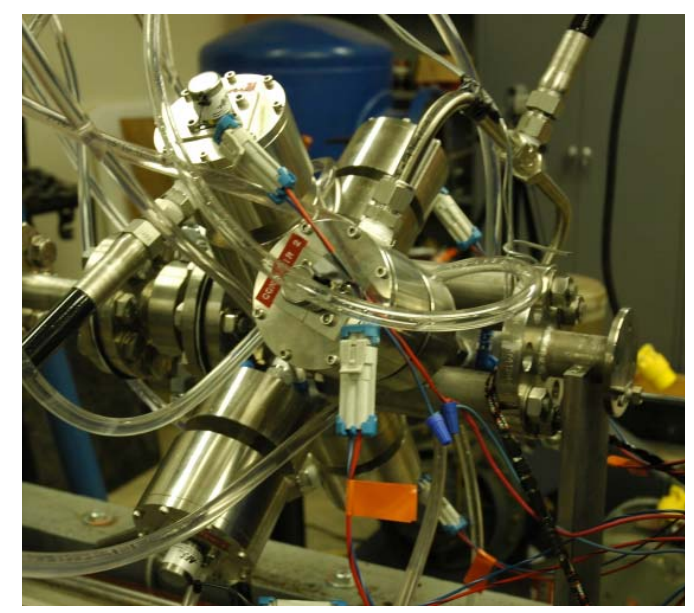

Fig. 3 Photograph showing gas injector housing and water cooling subsystem mounted on the PDE platform.

design of the detonation tube, optimization of the gas injector timing as well as the use of additional gas injectors operating out-of-phase.

Returning to the experimental setup, the gas injector opening and closing timing was adjusted by modulating the control signal pulse-width to vary the propane, oxygen and purge air injection volumes. Since the gas injectors were intended for use in internal combustion engines with lower working temperatures and pressures than those encountered in PDEs, check valves and flash arrestors located between the gas injectors and the injection system were used to prevent any shock waves from propagating toward them. Furthermore, the gas injectors were isolated from the rapid temperature rise during pulsed detonation operations by incorporating customdesigned water-cooling subsystems between them and the detonation tube (see Figs. 2 and 3).

\section{Propane, oxygen and purge air delivery}

Propane and oxygen were delivered to the gas injectors via standard $12.7 \mathrm{~mm}(1 / 2 \mathrm{in}$.) stainless steel tubes from the storage cylinders, while purge air was delivered from a high-pressure compressor. Their working pressures were metered using individual pressure gauges prior to their entry into the detonation tube. Since accurate operation of the gas injectors required accurate metering of the gaseous working pressures and the programmed control pulse widths, no attempts were made to calibrate the flow rates of all the gaseous flows. However, due to the design of the gas injectors which required circumferential inflow of the gases, custom-designed housings were used to house the gas injectors and to provide the required inflow conditions. The gases, after leaving the gas injectors, passed through check valves before finally exhausting into the injection section of the detonation tube. The experiments were all conducted with stoichiometric propane-oxygen mixtures at initial conditions of one atm and $20^{\circ} \mathrm{C}$. Flexible hoses for the final flow delivery were used to minimize installation interference with the force measurement. It is worth noting that at the prescribed experimental conditions, the detonation cell size $(\lambda)$ of stoichiometric propane and oxygen mixture is estimated to be $1.3 \mathrm{~mm}$. Furthermore, it is generally accepted that a minimum tube diameter of $D / \lambda=13$ is required for successful detonations propagation. Hence, based on the inner diameter of the present detonation tube, the tube diameter is approximately $D / \lambda=18.7$ which exceeded the minimum tube diameter significantly.

\section{Data acquisition and system control}

A National Instruments PXI data acquisition system capable of high-speed simultaneous data operations was used for both system control and data acquisition. The hardware was integrated with LabView ${ }^{\mathrm{TM}}$ to control the entire test and data acquisition process. Time-accurate TTL signals from the system were used to trigger and operate all the gas injectors. Signal outputs from the six PCB 111A24 pressure transducers and the PCB $201 \mathrm{~B} 05$ load cell were acquired at 16-bit resolution. The pressure transducers and the load cell all had bandwidths of 1 MHz. The pressure transducers were mounted along the detonation measurement section while the load cell was mounted against a rigid support near the injection section. Output from the transducers and the load cell were directed to a PCB 483A signal conditioner and then to the data acquisition system as seen in the upper right of the schematic of the system connectivity in Fig. 4. Simultaneous sample-and-hold of the signals was performed at 240 


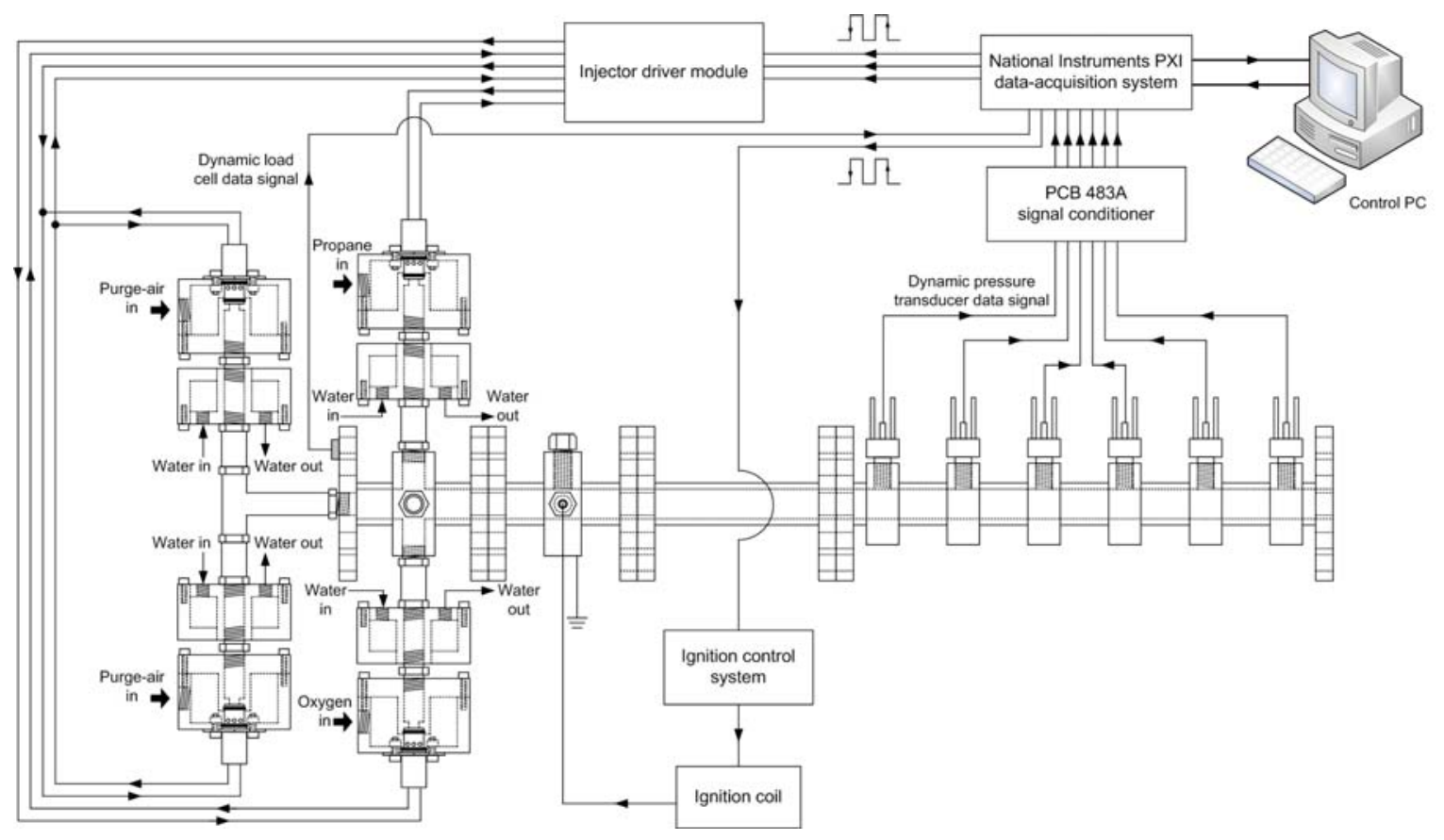

Fig. 4 Schematic of the full system connectivity for the PDE platform.

$\mathrm{kHz}$ for $5 \mathrm{~s}$ periods during each experiment. The time-resolved pressure data allowed for the calculations of wave velocities using the time-of-flight method.

As for system control, in particular the gas injectors, digital high-low TTL signals from the data acquisition system were channeled into the injector driver module to control the opening and closing operations. The opening and closing operations included appropriate time delays within the program routines to take into consideration the time responses of the various system components. Data transfer and control between the data acquisition system and the host PC were made through a fiber-optic link.

\section{E. DDT enhancement devices}

In addition to the "clean-tube" configuration without any DDT enhancement devices, several conventional as well as non-conventional configurations of DDT enhancement devices were studied. Shown in Fig. 5, these DDT devices include, first, a conventional helical Shchelkin spiral of 50\% blockage ratio which fitted against the inside wall of the detonation tube. The $160 \mathrm{~mm}$ long, $4 \mathrm{~mm}$ diameter spiral (L/D=6.6) with an $8 \mathrm{~mm}$ pitch was made from high-strength stainless steel (see Fig. 5(a)). Previous investigations ${ }^{17}$ using similar spirals but with $\mathrm{L} / \mathrm{D}=12.5$ succeeded in promoting DDT. However, as one of the aims of the present study was also to keep the size of the PDE system as compact as possible, an almost 50\% reduction in the allowable DDT section length and, hence, spiral length, was made in the expectation that successful detonations could still be attainable. Secondly, circumferential and helical groove configurations of zero blockage-ratio are used. These grooves are as long as the Shchelkin spiral. However, the grooves were designed so that they were machined into the walls of larger steel pipes. The inner diameters of these two groove configurations were similar to the bare detonation tube, that is, the diameter is 24.3 $\mathrm{mm}$, resulting in a zero blockage ratio with respect to the detonation tube dimensions. The width, depth and pitch of the grooves in both configurations were kept at 5, 4.55 and $10 \mathrm{~mm}$ respectively for consistency, with the only difference being in the arrangement of the grooves (see Fig. 5(b)-(c)). It should be noted here that for the circumferential groove configuration, the grooves did not actually circumscribe the full circumference for obvious structural reasons. Lastly, two-dimensional, convergent-divergent throat configurations were also included for DDT enhancement testing. As with the Shchelkin spiral, their blockage-ratios were also kept at $50 \%$ for consistency's sake. Despite sharing the same blockage ratio, the two configurations possessed symmetrically-arranged 15 and 30 deg convergent-divergent throat designs as shown in Fig. 5(d)-(e). The throats were located along the centerline of a substantially shorter DDT section, $60 \mathrm{~mm}$ long, since the throat designs did not depend on the DDT section length, unlike the Shchelkin spiral and the grooves. The use of the convergent-divergent throats in the present study was prompted by the possibility of producing a sufficiently powerful Mach stem arising from the coalescence of the incident and reflected shocks around the convergent section to induce successful detonations. The two different 


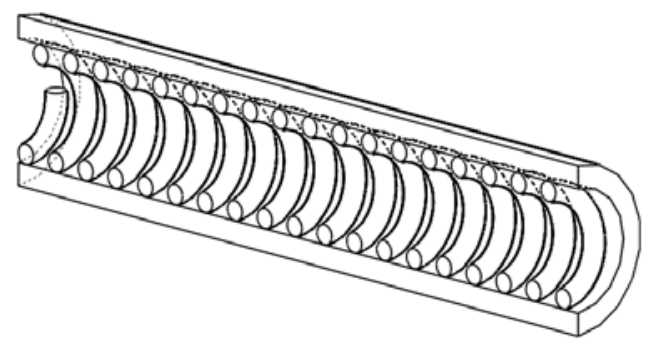

(a) Shchelkin spiral

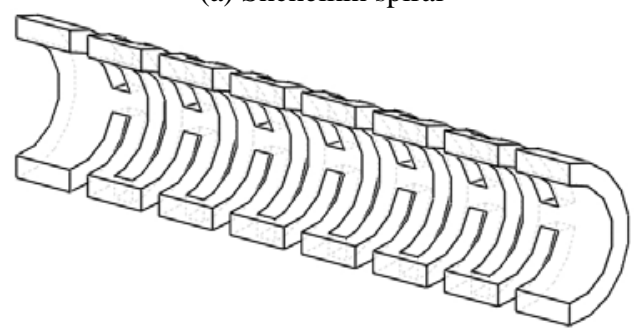

(b) Circumferential grooves

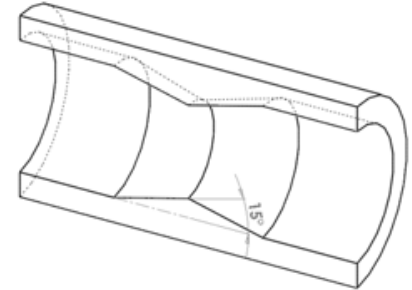

(d) 15 deg convergent-divergent section
Fig. 5 Design schematics of DDT enhancement devices.

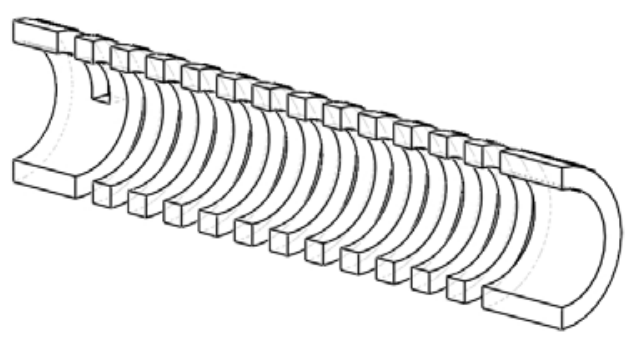

(c) Helical grooves

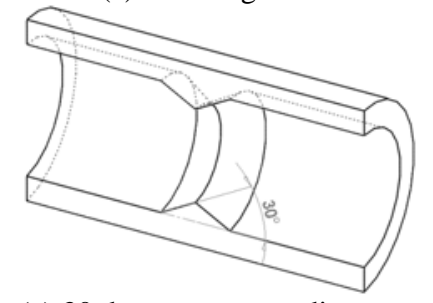

(e) 30 deg convergent-divergent

convergent-divergent angles were studied to determine whether significant differences would arise due to angular variation. Moreover, the two convergent-divergent throats were also combined and tested during the study to see if any significant differences existed between each throat configuration.

\section{Results and Discussion}

The experimental study began with a series of tests with firing frequencies from 10 through $30 \mathrm{~Hz} \mathrm{at} 5 \mathrm{~Hz}$ intervals to verify the reliability and robustness of integrating gas injectors into the PDE platform. Subsequently, 15 $\mathrm{Hz}$ was selected as the common operating frequency for testing the DDT enhancement devices. With further improvements to the system design and controls anticipated in the future, the firing frequency can be increased to match the maximum injection frequency of the gas injectors. However, as a limited initial assessment of utilizing the commercial gas injectors for PDE operations, the $15 \mathrm{~Hz}$ operating frequency should suffice.

\section{A. Detonation success-rates}

To evaluate whether successful detonations occurred and how regular they occurred as a percentage of the total number of firings, both time-of-flight velocities and pressure levels across all six pressure transducers were used to assess each and every firing. The velocities obtained by the time of flight method were calculated by dividing the distance between two pressure transducers by the time of passage of the pressure peaks, while peak pressure levels registered by all the pressure transducers were used. A firing is deemed to lead to a successful detonation only if both the average TOF velocity and peak pressure across the six pressure transducers exceed theoretical ChapmanJouguet (CJ) velocity and pressure for stoichiometric propane-oxygen detonations. In the present study, the CJ detonation velocity and pressure are $2360 \mathrm{~m} / \mathrm{s}$ and 36.7 bars respectively. Figure 6 shows the detonation successrates for all the DDT enhancement devices for stoichiometric propane-oxygen initially at room conditions. Surprisingly, clean tube configuration without any DDT devices registered the highest success-rate with $76 \%$ of the firings reaching detonation conditions successfully, followed by the Shchelkin spiral configuration with a $70 \%$ success rate. Configurations utilizing circumferential and spiral grooves are next with success rates of approximately $61 \%$ and $48 \%$ respectively. Finally, convergent-divergent throats achieved significantly lower success rates at $35 \%$, $24 \%$ and $17 \%$ for the $30+15$ deg C-D throat combination, the 15 deg C-D and the 30 deg C-D throats respectively. These results revealed a highly intriguing and counter-intuitive observation whereby any DDT enhancement device 


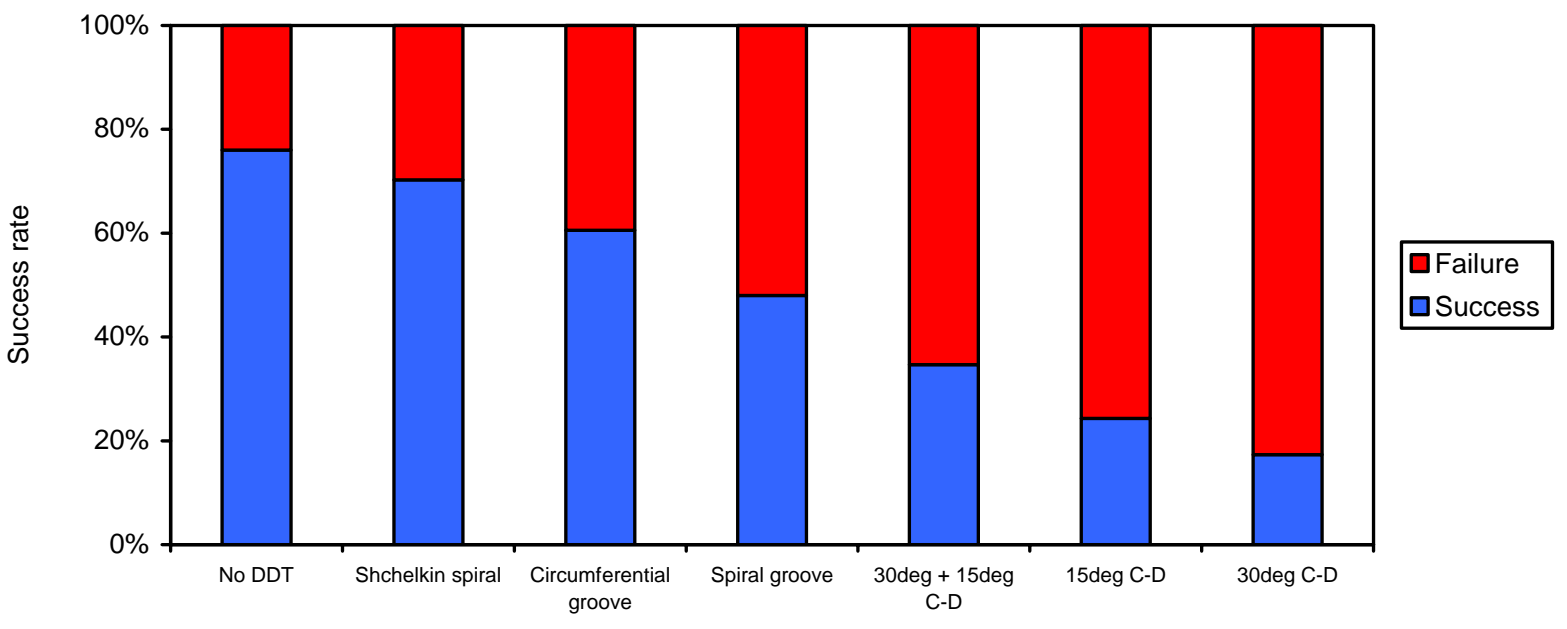

DDT-enhancement devices

Fig. 6 Detonation success rates for the investigated DDT-enhancing devices.

used in the present PDE system would lead to a reduction in the detonation success rate, the extent of reduction being dependent on the exact type of device used.

The above results prompted two immediate questions: First, why did the use of DDT enhancement devices in the present PDE system fail to achieve detonation success rates higher than the clean-tube configurations, contrary to their intended purposes? And secondly, why did convergent-divergent throat configurations achieve the lowest success rates out of the six DDT-enhancement devices used? To answer these questions, it should be recalled in particular here that the length-to-diameter ratio of the Shchelkin spiral used in the present study measured approximately 6.6, which was significantly shorter than the corresponding spiral $(\mathrm{L} / \mathrm{D}=12.5)$ used in an earlier, successful study on DDT enhancements. ${ }^{17}$ However, as the current results demonstrated, a shorter-than-optimal spiral length not only failed to enhance the DDT phenomenon but, instead, degraded the detonation success rate to a level lower than if it was not used at all. Based on similar arguments, it is reasonable to assume that conceptuallysimilar groove configurations would also suffer similar problems. Furthermore, their even lower success-rates as compared to the Shchelkin spiral may be due to the poorer mixing and/or filling of propane and oxygen caused by the grooves. It is not inconceivable that pockets of propane/oxygen/detonation products were more likely to be trapped along some sections of the grooves, especially when the zero blockage ratio grooves were actually designed such that they could be considered to be embedded within the inner tube wall.

As for the second question, the results showed that the detrimental influence of the DDT enhancement devices can be broadly divided into two groups: One group comprises of Shchelkin spiral, circumferential grooves and spiral grooves and the other group comprises of the convergent-divergent throat configurations. The first group incidentally consists of configurations which achieved the highest detonation success rates (though not exceeding the baseline clean-tube configuration) while the second group achieved the lowest detonation success rates. Upon closer inspection, one can observe that the configurations in the first group are conceptually similar with the intention to enhance DDT through the presence of three-dimensional undulations along the detonation tube walls to promote flame turbulence, differences in the blockage-ratio notwithstanding. In contrast, configurations in the second group attempted to produce Mach stems to promote successful detonation using two-dimensional convergent-divergent throat designs (see Fig. 5). Although the blockage ratio may be similar for the Shchelkin spiral and the three convergent-divergent configurations, the latter configurations were likely to pose a more serious flow blockage as their designs were two-dimensional. Unlike three-dimensional undulations used in the Shchelkin spiral where the continuously spiraling design might prove easier for the injected propane and oxygen to flow and mix within the detonation tube, strictly two-dimensional physical blockages such as these convergent-divergent throats would prove difficult for the propane and oxygen injections to mix and fill up the detonation tube properly. Instead of producing Mach stems to promote successful detonations, the throats might have constricted the proper flows of propane and oxygen throughout the entire detonation tube during their injection phases. In fact, it is not impossible for the throats to even adversely influence the actual flame and shock front couplings. 


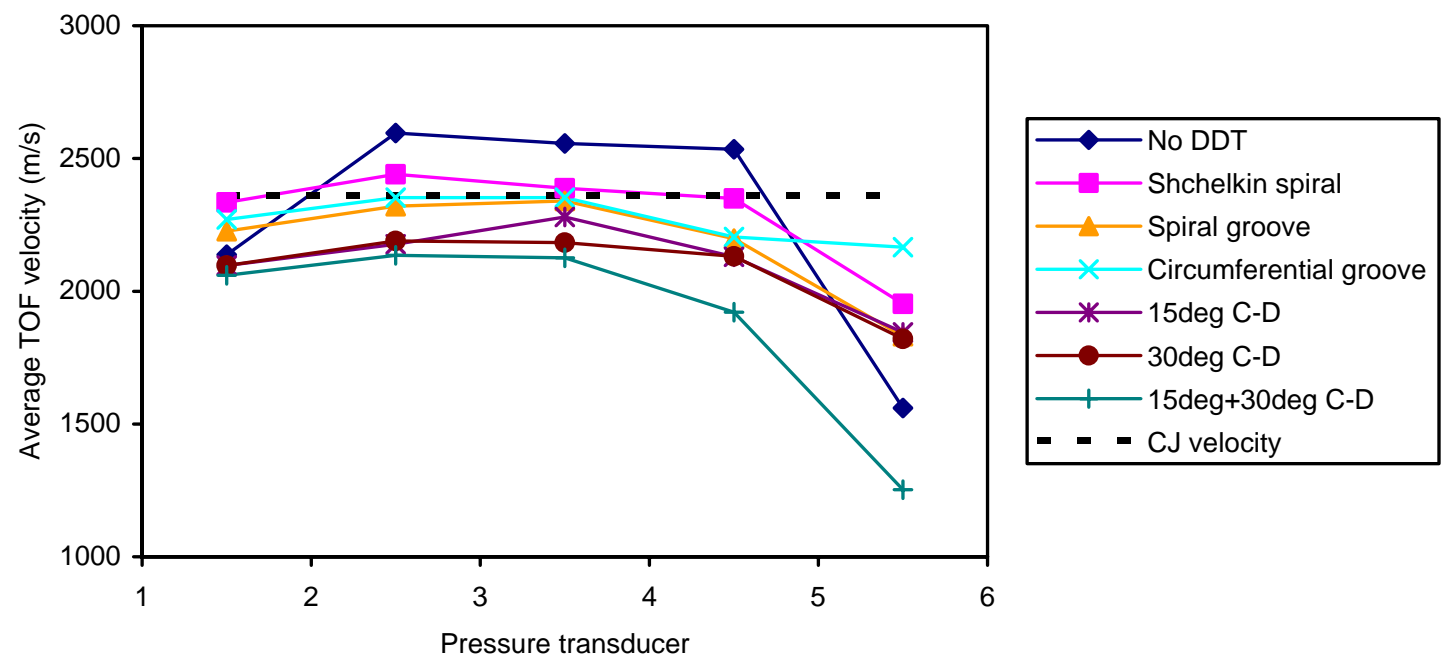

Figure 7. Average TOF velocity profiles of deflagrations which failed to transit to detonations successfully.

To probe these issues further, averaged TOF velocities from unsuccessful firings for each device are shown in Fig. 7 to shed some light on how they failed. In Fig. 7 where the CJ velocity is shown for reference, it can be observed that the average TOF velocities generally increased between the first and third pressure transducer locations for most of the test cases. However, the average TOF velocities gradually decrease thereafter until they were drastically reduced near the detonation tube exit. Whether the sudden reduction in the TOF velocities near the tube exit was due to slightly-imperfect filling of the tube or the influence of the tube exit on the deflagration/detonation behavior remains unclear, although successful detonation results showing similar behavior seems to indicate the latter explanation is more probable. More significantly, the figure shows that the average TOF velocities for failed detonations could still exceed the CJ velocity level even if their corresponding pressures did not reach CJ pressure, particularly for the clean-tube and Shchelkin spiral configurations. This is likely to be caused by the imperfect coupling between the flame and shock fronts which would otherwise increase the peak pressure levels drastically to qualify as successful detonations. On the other hand, it is also possible that the data-acquisition system's $240 \mathrm{kHz}$ sampling rate leads to appreciable errors in the pressure measurements. All the other configurations failed to achieve average TOF velocities above the CJ velocity although it is worth mentioning here that these average velocities stayed above $2000 \mathrm{~m} / \mathrm{s}$ for most of the detonation tube length, except for locations near the tube exit. Either the DDT enhancement devices used in the present study might just be a little too short for them to effectively promote the DDT phenomenon successfully, or that the propane-oxygen mixture did not manage to fill the detonation tube entirely.

In contrast, Fig. 7 reveals that the average TOF velocities for the convergent-divergent throat configurations are the lowest among the failed cases. Although highly speculative at this stage, these results also lends support to the earlier conjecture that the throats might have prevented more successful detonations by significantly disrupting the continued flame and shock front couplings and perhaps even slowing down the flame fronts due to their present geometric design.

\section{B. Dynamic pressure measurements}

To further understand the behavior of the successful detonations as well as the influence of the various DDT enhancement devices on the evolution of the detonation pressures, Fig. 8 shows typical dynamic pressure profiles from the six pressure transducers of successful detonations for clean-tube and Shchelkin spiral configurations. Both figures revealed largely similar behavior where the peak pressure levels are usually quite high with occasional fluctuations, although there remained some definite differences upon closer inspection. For instance, in the case of the clean-tube configuration, the peak pressure started out very high at approximately 74 bar at the first pressure transducer location but reduced to 45-60 bar thereafter (except at pressure transducer location four which could possibly result from poor data resolution). A general decline in the peak pressure level can be discerned from Fig. 8(a) even though this particular example reached successful detonation conditions both in terms of the TOF velocities and peak pressures. On the other hand, the Shchelkin spiral configuration appears to maintain the peak 


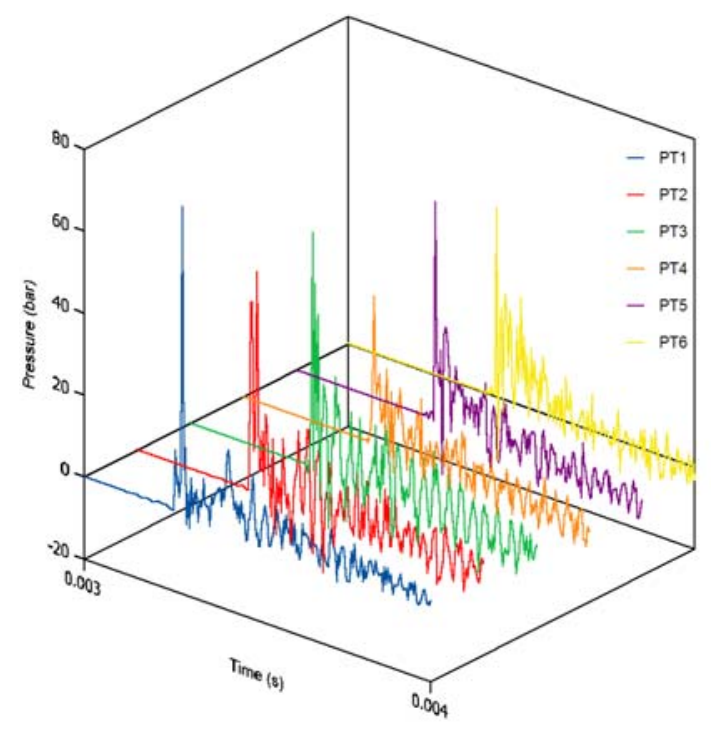

(a) Clean-tube

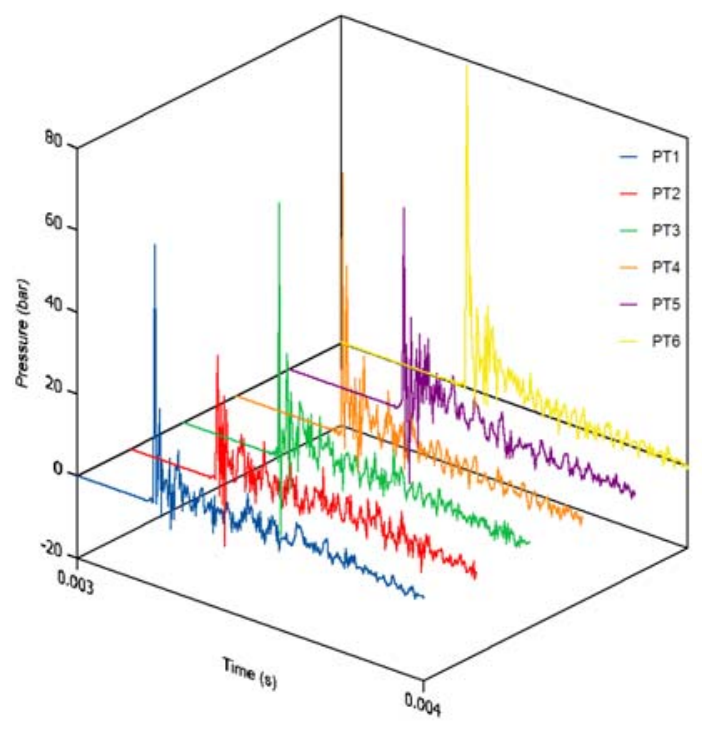

(a) Shchelkin spiral

Fig. 8. Dynamic pressure profiles for successful detonations using clean-tube and Shchelkin spiral configurations

pressure levels better than the clean-tube configuration, except for the occasional pressure fluctuations which could be due to the inability of the data-acquisition system to capture the von Neumann spike. Similar to the clean-tube configuration, peak pressure levels of the detonation front reached a high of approximately 60 bar before fluctuations set in. Surprisingly though, the last pressure transducer registered a sudden increase in peak pressure which almost hit 80 bar. This erratic peak phenomenon could be due to the difficulty of resolving the sharp pressure peaks that are narrower than the transducer diameter and by the limited sampling rate of the data-acquisition system. Nonetheless, the pressure profiles generally resemble what would be to be expected for a successful detonation and were verified earlier by the associated TOF velocities.

Another surprising aspect regarding the above results is that the peak pressure levels shown in Fig. 8 are quite high, typically ranging between 40-60 bar, despite using a relatively modest $150 \mathrm{~mJ}$ rated ignition system. This observation is in agreement with the study carried out by Cooper et al. ${ }^{14}$ where they achieved $40-50$ bar peak pressure levels in single-shot DDT-enhanced detonation experiments using only a $50 \mathrm{~mJ}$ ignition system. This is further supported by another single-shot experimental detonation study by $\mathrm{Li}$ et al. ${ }^{18}$ using similar propane-oxygen mixtures which showed peak pressure variation of 40-120 bar, although the ignition energy used by them was more than three times that used here ( $470 \mathrm{~mJ}$ compared to $150 \mathrm{~mJ}$ ).

On the other hand, Fig. 9 shows the pressure profiles for unsuccessful detonations using the helical groove and the $30+15$ deg convergent-divergent throat configurations to highlight how similar devices might have failed to set off successful detonations. As can be observed in Fig. 9(a), the peak pressure levels remain approximately between 30-40 bar with a declining trend towards the detonation tube exit, with the exception of a sudden increase to 84 bar at the third pressure transducer location. Even if this sudden increase signifies an almost successful transition from the borderline peak pressure levels (since detonation pressure is assumed to be 36.7 bar), it is clearly not sustainable even at the next pressure transducer location shortly downstream. Coupled with average TOF velocities lower than the CJ velocity, this indicates that the helical grooves did not have as much ability as the Shchelkin spiral to accelerate the flame fronts sufficiently for detonations to occur. As for the $30+15$ deg convergent-divergent throat configuration, the average TOF velocities did not manage to reach the CJ velocity although the peak pressure levels registered relatively high values at between 40-90 bar. Most interestingly, one can observe that despite registering well-defined pressure peaks at the first five pressure transducers, the last pressure transducer shows the pressure profile to possess two well-defined pressure peaks instead. The difference between these two pressure peaks is approximately 20 bar (first and second peak pressure at approximately 60 and 80 bar respectively) but as the peak pressure levels are quite high to begin with, it appears that they signify a significant decoupling of the flame and shock fronts. Although it remains difficult to judge confidently from the available results, this evidence agrees well with the earlier postulate that the use of the prescribed convergent-divergent throat configurations in the present setup might lead to decoupling of the fronts, which is contrary to the envisaged intention of actually reinforcing the coupling between the fronts. 


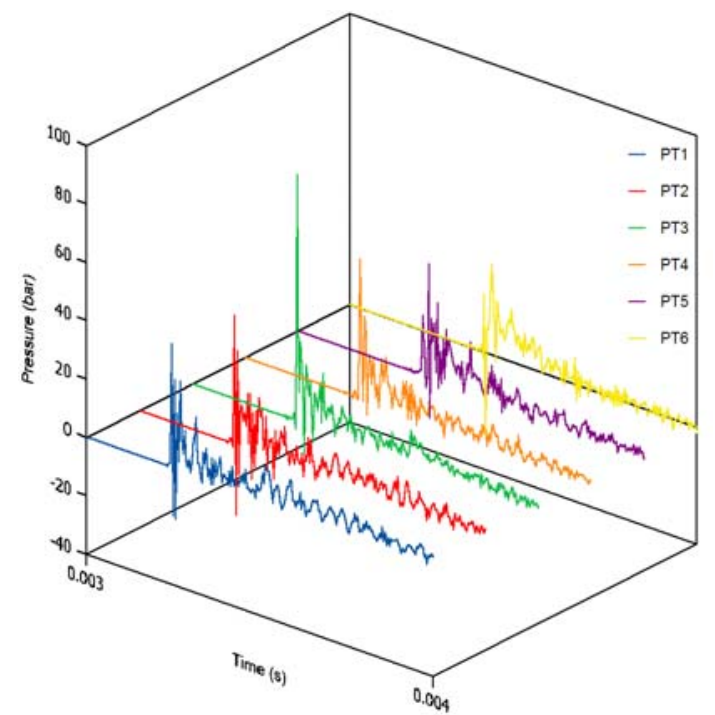

(a) Helical groove

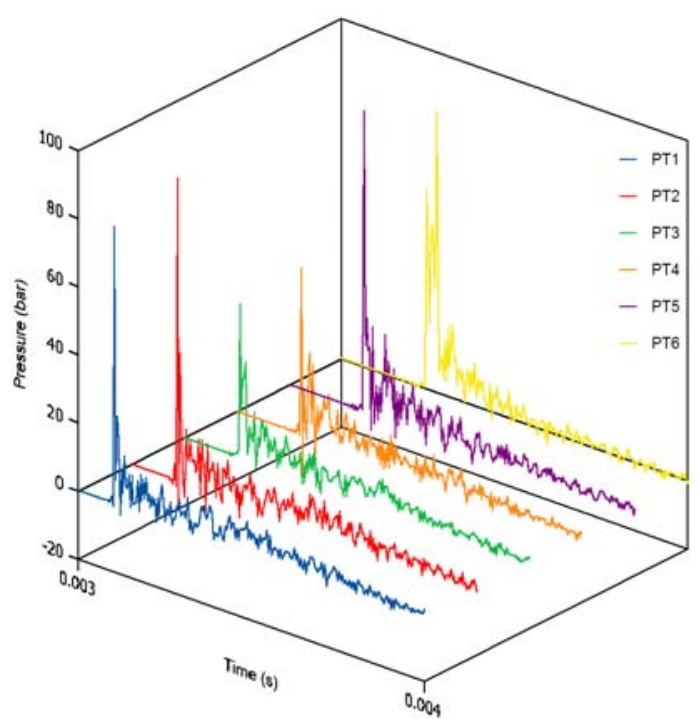

(b) 30 deg +15 deg convergent-divergent throat

Fig. 9. Dynamic pressure profiles for unsuccessful detonations using helical groove and $30 \mathrm{deg}+15 \mathrm{deg}$ convergent-divergent throat configurations.

It is also interesting to note that the peak pressure levels occasionally exceed the CJ pressure for stoichiometric propane-oxygen mixtures (see Fig. 8 and 9), which seems to indicate that overdriven detonations occurred at times during the present study. The exact reasons for such occurrences remained unclear since the ignition energy level appears to be too low, in particular for the clean-tube configuration where no DDT-enhancing devices were used.

\section{Operational issues}

During the course of the investigation, attention was paid toward any potential operational problems, in particular those arising from the use of internal combustion engine gas injectors in a PDE platform. One of the biggest motivations for using gas injectors is that they are able to provide time-accurate propane-oxygen mixture injections as shown in Fig. 10, where the time intervals between injections can be maintained virtually constant. This represents a significant improvement over rotary valve injection mechanisms where irregular timings between consecutive detonations are not unexpected. However, the figure also shows a recurring problem associated with this study. It can be observed that significant variations in the peak pressure level exist with the minimum peak pressure levels as low as half of those associated the maximum pressure levels. Although it could be the case that some deflagrations might not have successfully transitioned into detonations, the possibility that the variations were caused by variations in gas injector response, leading to non-

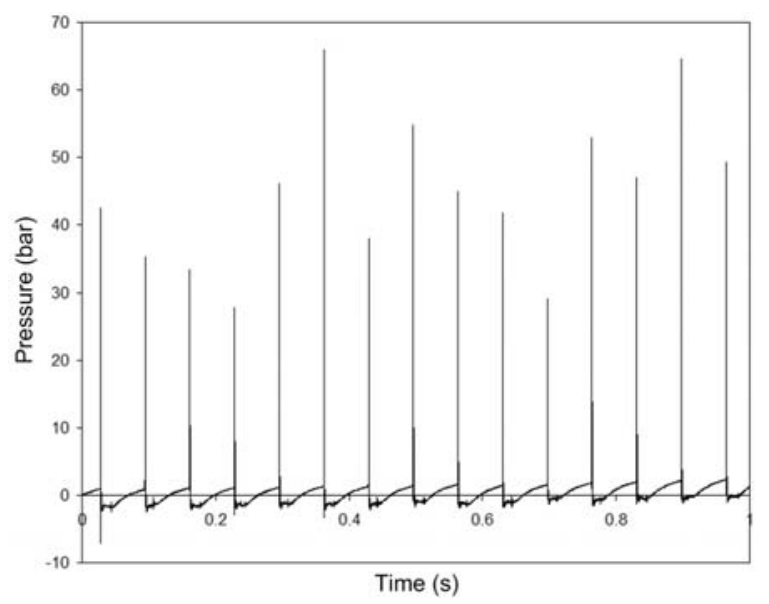

Fig. 10. Fluctuations of the peak pressure levels over a one-second firing period with Shchelkin spiral. optimal filling or mixing of the detonation tube prior to ignition cannot be ignored. When the operations of the gas injectors are dictated by millisecond triggering signal pulse-widths, seemingly small discrepancies in the response times of each gas injector may accrue into significant deviations from the expected behavior. Although there will definitely be uncertainties involved during actual pulse detonation operations, it may be worthwhile to fine tune all gas injectors "in-situ" prior to the actual experiments to minimize experimental errors. Another possible explanation for the above observation could be that the sampling frequency of the data acquisition system might not be sufficiently high to fully resolve the data. To fully resolve the one microsecond response time signals from these pressure transducers to reduce uncertainties in the captured data, a sampling rate of at least $1 \mathrm{MHz}$ per pressure 
transducer would be required, which proved too high for the data acquisition system to manage reliably during the study. These possible sources of experimental uncertainties will be explored in detail in a future study.

\section{Conclusions}

An experimental investigation on the use and integration of commercial off-the-shelf gas injectors for studying nonconventional DDT enhancement devices has been carried out. Although the gas injectors are meant for use in internal combustion engines, the study showed that they performed satisfactorily and may be proved to be adequately robust for prolonged pulse detonation operations (attempts to carry out prolonged testing are being planned), provided sufficient measures are taken to mitigate potential operating hazards. The injectors were found to be capable of delivering pulsed gas flows accurately and were superior to rotary-valve based injection systems. The DDT enhancement device test cases were studied at a firing frequency of $15 \mathrm{~Hz}$ and TOF velocity results showed that clean-tube configuration was surprisingly able to achieve the highest overall detonation success rate as compared to the use of DDT-enhancement devices. This demonstrates that in the design of compact PDE systems, considerable efforts are required to optimize the DDT device configurations. Despite this, Shchelkin spiral is observed to be the best performer among the DDT enhancement devices, followed by groove configurations and, lastly, convergent-divergent throat configurations. From the pressure measurements, one possible reason why the groove configurations did not work well is that they were designed with zero blockage-ratio which reduced the production of flame turbulence. The design might have also led to increased trapping of unburned reactants or products which reduce the effectiveness of the grooves to promote flame turbulence. On the other hand, results suggest that convergent-divergent throat configurations have a tendency to destabilize the coupling between the flame and shock fronts. Lastly, it was observed that significant variations in the peak pressure levels existed in the pressure measurements which could be due to inconsistencies in the gas injector response times, partial success in the DDT phenomenon or insufficient sampling rate of the data acquisition system and will be addressed in future studies.

\section{Acknowledgments}

The authors would like to acknowledge the research funding provided by the Singapore Defense Science and Technology Agency for the study, technical assistance in carrying out the experiments by K. S. Lim of Temasek Laboratories, as well as data analysis and insights provided by J. M. Li of National Cheng Kung University, Taiwan. Useful discussions with Y. T. Jiang of DSO National Laboratories and their support for the project are also appreciated.

\section{References}

${ }^{1}$ Cambier, J. L., and Tegner, J. K., “Strategies for Pulsed Detonation Engine Performance Optimization,” Journal of Propulsion and Power, Vol. 14, No. 4, 1998, pp. 489-498.

${ }^{2}$ Jackson, S. I., and Shepherd, J. E., “Initiation Systems for Pulse Detonation Engines,” AIAA Paper 2002-3627, 2002.

${ }^{3}$ Fan, W., Yan, C., Huang, X., Zhang Q., and Zheng, L., "Experimental Investigation on Two-Phase Pulse Detonation Engine,” Combustion and Flame, Vol. 133, No. 4, 2003, pp. 441-450.

${ }^{4}$ Kailasanath, K., "Recent Developments in the Research on Pulse Detonation Engines,” AIAA Journal, Vol. 41, No. 2, 2003, pp. 145-159.

${ }^{5}$ Allgood, D., and Gutmark, E., "Performance Measurements of Multi-Cycle Pulse Detonation Engine Exhaust Nozzles," AIAA Paper 2005-0222, 2005.

${ }^{6}$ Allgood, D., and Gutmark, E., “Performance Measurements of Pulse Detonation Engine Ejectors,” AIAA Paper 2005-0223, 2005.

${ }^{7}$ Tangirala, V., “Performance Estimations of a Pulse Detonation Engine with Nozzles,” AIAA Paper 2006-4792, 2006.

${ }^{8}$ Ebrahimi, H., "Numerical Investigation of 2-D and 3-D Multitube Pulse Detonation Using $\mathrm{H}_{2}$ and JP8 Fuel," AIAA Paper 2004-0465, 2004.

${ }^{9}$ He, X., and Karagozian, A. R., "Pulse-Detonation-Engine Simulations with Alternative Geometries and Reaction Kinetics," Journal of Propulsion and Power, Vol. 22, No. 4, 2006, pp. 852-861.

${ }^{10}$ Kenfield, J. A. C., “Thermodynamics of Air-Breathing Pulse Detonation Engines,” AIAA Paper 2001-3982, 2001.

${ }^{11}$ Heiser, W. H., and Pratt, D. T., “Thermodynamic Cycle Analysis of Pulse Detonation Engines,” Journal of Propulsion and Power Vol. 18, No. 1, 2002, pp. 68-76.

${ }^{12}$ Hutchins, T. E., and Metghalchi M., "Energy and Exergy Analyses of the Pulse Detonation Engine," ASME Journal of Engineering for Gas Turbines and Power, Vol. 125, No. 4, 2003, pp. 1075-1080.

${ }^{13}$ Wu, Y., Ma, F., and Yang, V., "System Performance and Thermodynamic Cycle Analysis of Airbreathing Pulse Detonation Engines,” Journal of Propulsion and Power, Vol. 19, No. 4, 2003 pp. 556-567. 
${ }^{14}$ Cooper, M., Jackson, S., Austin, J. M., Wintenberger, E., and Shepherd, J. E. “Direct Experimental Impulse Measurements for Detonations and Deflagrations,” Journal of Propulsion and Power, Vol. 18, No. 5, 2002, pp. 1033-1041.

${ }^{15}$ Lee, S. Y., Watts, J., Saretto, S., Pal, S., Conrad, C., Woodward, R., and Santoro, R., "Deflagration to Detonation Transition Processes by Turbulence-Generating Obstacles in Pulse Detonation Engines,” Journal of Propulsion and Power, Vol. 20, No. 6, 2004, pp. 1026-1036.

${ }^{16}$ Lu, F. K., Meyers, J. M., and Wilson, D. R., "Experimental Study of a Pulse Detonation Rocket with Shchelkin Spiral," 24th International Symposium on Shock Waves, Beijing, China, 2004, Paper No. 1054.

${ }^{17}$ New, T. H., Panicker, P. K., Lu F. K., and Tsai, H. M., "Experimental Investigations on DDT Enhancements by Schelkin Spirals in a PDE,” AIAA Paper 2006-0552, 2006.

${ }^{18} \mathrm{Li}$, J., Chung, K., and Lai, W. H., “Overdriven Phenomena in Deflagration-to-Detonation Transition Process,” Aeronautical and Astronautical Society of the Republic of China Joint Conference, 2005. 